\title{
The Clarification of the Strategy during Sit-to-Stand Motion from the Standpoint of Mechanical Energy Transfer
}

\author{
Masaya AnAn, RPT, MSP, TAKUYA IBARA, RPT, MSP, \\ Nobuhiro Kito, RPT, PhD \\ 1) Department of Physical Therapy and Occupational Therapy Sciences, Graduate School of Health \\ Sciences, Hiroshima Unversity: 2-3-1 Kasumi, Minami-ku, Hiroshima-shi, Hiroshima 734-8553, \\ Japan.TEL:+81 82-257-5417,E-mail: anan@hiroshima-u.ac.jp \\ 2) Department of Rehabilitation, Kawashima Orthopaedic Hospital \\ 3) Department of Physical Therapy, Faculty of Health Sciences, Hiroshima International University
}

\begin{abstract}
Purpose] This study aimed to clarify the motion strategy used by young healthy adults during sit-tostand motion (STS) and to investigate the relationship between forward tilt movement by the trunk and shanks, which is considered to contribute to the forward shift of COG, and the load on the lower extremity joints accept, from the standpoint of mechanical energy flow. [Subjects] The subjects were 10 young healthy males without disease and/or present history of illnesses that would affect STS. [Methods] Three kinds of task conditions were adopted: normal STS (condition N), STS with the trunk tilted as forward as possible (condition TM), and STS with the trunk as vertical as possible (condition TV). The kinematic data during STS were collected using a three-dimensional motion analysis system, and the kinetic data were collected by force plates. [Results] The average negative power in the proximal portions of the shank under condition TV was significantly higher than that under conditions $\mathrm{N}$ and TM. [Conclusion] Shank forward tilt appears to be primarily a reactive movement facilitating knee extension rather than contributing to moving COG forward. Thus, less trunk forward tilt movement results in the requirement of higher ability of shank forward tilt movement.

Key words: Sit-to-stand, Energy flow, Moment power
\end{abstract}

(This article was submitted Sep. 21, 2011, and was accepted Oct. 24, 2011)

\section{INTRODUCTION}

Sit-to-stand (STS) is one of the motions routinely repeated every day. STS is a dynamic motion that requires extensive joint movement in the lower extremities and trunk, and it also subjects the lower extremity joints to a heavy load during the posture change from sitting to standing Moreover, STS is a complicated motion to carry out and has two components: (1) transition from a wide base of support (BOS) created by the buttocks, thighs and feet to a narrow BOS created only by the feet, and (2) lifting the center of gravity $(\mathrm{COG})$ from the height of sitting to that of standing 2,3 . Nyberg et al. 1 reported that many falls by elderly people occur during transfers, indicating that STS is a motion with a risk of falls for the elderly and/or physically disabled people. STS is associated with applied motion such as patient transfer and walking. Acquiring STS is an essential factor to improve the mobility capability.

Recently, the validity of STS strategies which exhibit the most efficient muscular activity and least momentum utilization are being discussed 6 . STS strategies include the force-control strategy, the momentum strategy, a strategy using the upper extremities and a combination of these strategies. The force-control strategy that elderly and/or disabled people tend to use requires the generation of a large amount of force. This is because in this strategy, the trunk moves forward after the initiation of motion, the COG is transferred onto the BOS created by the feet, and then the body is elevated vertically. Although this strategy emphasizes stability control, it is a less efficient approach which doesn't utilize momentum from a mechanical perspective. Thus, the force-control strategy utilizes force of the lower extremities. In contrast, the momentum strategy, generally adopted in young healthy adults, is the most efficient approach. This is because in this strategy, the momentum generated through the movement of trunk is transferred to the lower extremities, and the body smoothly moves on to the next posture without pausing. Thus, the momentum strategy utilizes the momentum of the upper half of the body. There is also a strategy which uses the upper extremities, which is executed by pressing the seat, armrest(s), and so on to ensure both stability and progression.

The ratio of mechanical energy to physiological energy generated by muscles during motion is defined as efficien-

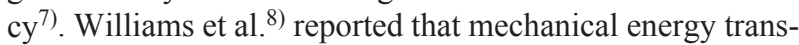
ferred between segments increases the efficiency of utilized physiological energy, and increase of mechanical energy transfer suppresses the physiological energy exertion by 
muscles, enhancing their efficiency. Yu et al. 1 p reported that in STS, knee and hip joints influence the upward velocity of COG, while the movements of the ankle and hip joints influence the forward velocity of COG. Thus, it appears that the forward tilt of the trunk and shanks contributes to the forward shift of COG, and these movements change the force distribution on each of the lower extremity joints, playing a role in effectively transferring energy through the actions of buttocks-off and extension of the lower extremity joints. In our literature searches, we could not find STS studies which had examined the actual mechanical flow of energy. Accordingly, this study aimed to clarify the motion strategy used by young healthy adults during STS and to investigate the relationship between forward tilt movement by the trunk and shanks considered to contribute to the forward shift of COG, and the load on the lower extremity joints accept, from the standpoint of mechanical energy flow.

\section{SUBJECTS AND METHODS}

The subjects were 10 young healthy males without disease and/or present history of illnesses that would affect STS (mean age $22.2 \pm 1.3$ years, mean height $173.2 \pm 5.2 \mathrm{~cm}$, mean weight $65.0 \pm 7.4 \mathrm{~kg}$, and mean BMI $21.7 \pm 1.9 \mathrm{~kg} /$ $\left.\mathrm{m}^{2}\right)$.

Prior to the experiment, the purpose of this study was thoroughly explained to the subjects and their oral and written consents were obtained. This study was undertaken with the approval of the Ethics Committee of the Division of Physical Therapy and Occupational Therapy Sciences, Graduate School of Health Sciences, Hiroshima University.

The ordinary sitting posture was used as the initial sitting posture. A chair with a height-adjustable seat without a backrest and armrests was used. The vertical distance between each subject's lateral knee joint space and the floor was used for the seat height. The shanks were maintained vertical. Subjects were barefoot, and the width separating their feet was set as the distance between the anterior superior iliac spines. The depth for sitting was set so that the midpoints between the greater trochanter and lateral epicondyle of the femur were aligned with the front edge of the seat. The subjects were instructed to face forward while sitting and to fold their arms on their chest to avoid masking the anatomical markers.

The task was STS from the initial sitting posture. Subjects started the motion at their own discretion after the examiner's oral cue. The motion speed was also performed at subject's discretion. Prior to measuring the motion, subjects practiced the motion sufficiently. The motion was measured three times. Three kinds of task conditions were used: normal STS (condition N), STS with the trunk tilted as forward as possible (condition TM), and STS with the upright trunk as vertical as possible (condition TV).

The kinematic data during STS were collected using Vicon MX, a three-dimensional motion analysis system (Vicon, Oxford, UK) with six infrared cameras. The kinetic data were collected by four force plates (Tec Gihan, Kyoto, Japan).

Infrared-reflecting markers $14 \mathrm{~mm}$ in diameter were at-
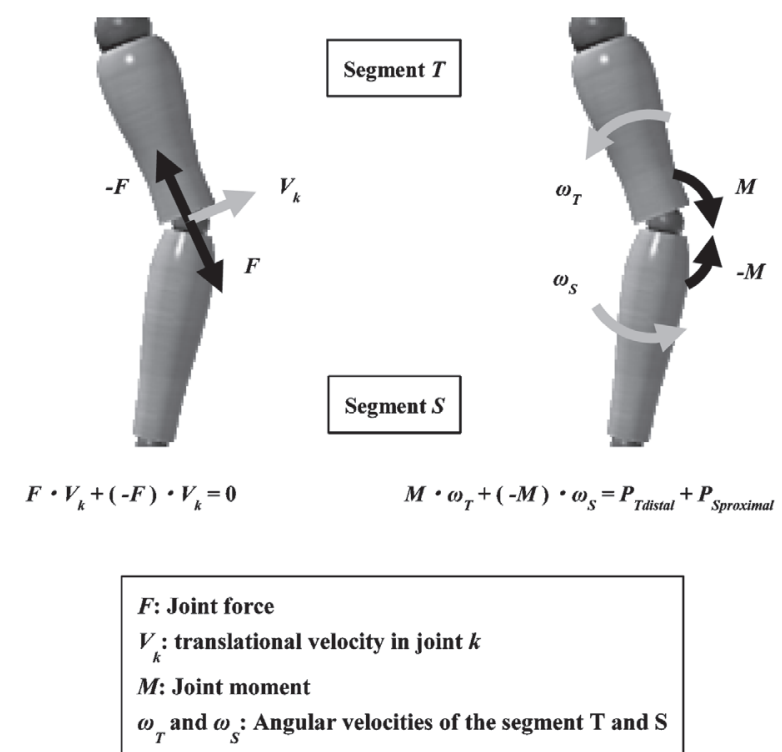

Fig. 1. Schematic diagram of energy transfer occurring between segments.

a) We assumed that the joint force transfers energy from one segment to the other but does not influence the total mechanical energy of the body.

b) Only the moments of force across joints are related to the increase and decrease of total mechanical energy.

tached to 40 landmarks: a pair at the temples, lateral ends of the superior nuchal line, tragus, acromion, olecranon, styloid process of the ulna, inferior edge of the last rib, superior edge of the iliac crest, anterior superior iliac spine, posterior superior iliac spine, great trochanter, lateral and medial epicondyles of the femur, lateral and medial condyles of the tibia, lateral and medial malleoli, head of the first and fifth metatarsal heads, and the calcaneal tuberosity. The spatial movements of the markers were captured by the three-dimensional motion analysis system at a sampling rate of 100 frame/s. At the same time, the three-dimensional ground reaction forces were collected by the force plates at a sampling frequency of $1,000 \mathrm{~Hz}$.

The coordinates of joint centers were calculated according to methods described in previous studies ${ }^{10,11}$. The ankle joint center was defined as the midpoint between the markers for lateral and medial malleoli of the ankle joint. The knee joint center was defined as the midpoint between the markers for lateral and medial epicondyles of the femur. For determining the hip joint center, a point one third of the distance between markers on the greater trochanter and the anterior superior iliac spine was first determined bilaterally. Then, a line was drawn connecting these points. The points $18 \%$ medial to the ends of this line were defined as hip joint centers. The abdomen-pelvis center was defined as the midpoint between the superior edges of the bilateral iliac crests. The thorax-abdomen center was defined as the midpoint between the inferior edges of the lowest ribs. A rigid-body link model consisting of nine segments, thorax, abdomen, pelvis, both thighs, both shanks, and both feet, was constructed 
Table 1. Maximum forward tilt angles in each segment under the different conditions

\begin{tabular}{lccc}
\hline & Condition N & Condition TV & Condition TM \\
\hline Thorax [deg] & $57.0 \pm 8.6^{*}$ & $35.1 \pm 8.2^{\S}$ & $81.5 \pm 4.7$ \\
Pelvis [deg] & $30.1 \pm 13.9$ & $25.5 \pm 8.3^{\S}$ & $39.6 \pm 13.9$ \\
Shank [deg] & $11.7 \pm 2.2$ & $13.0 \pm 3.0$ & $14.2 \pm 4.7$ \\
\hline mean \pm standard deviation, $*$. vs Conditon TV $(\mathrm{p}<0.05)$, . vs Condition $\mathrm{TM}(\mathrm{p}<0.05)$
\end{tabular}

mean \pm standard deviation, *: vs Conditon TV $(\mathrm{p}<0.05)$, : vs Condition TM $(\mathrm{p}<0.05)$

Table 2. Joint moment impulses in each segment under the different conditions

\begin{tabular}{llcc}
\hline & Condition N & Condition TV & Condition TM \\
\hline Hip extension $[\mathrm{Nm} \cdot \mathrm{s} / \mathrm{kg}]$ & $0.93 \pm 0.27^{\S}$ & $0.71 \pm 0.32^{\S}$ & $1.55 \pm 0.46$ \\
Knee extension $[\mathrm{Nm} \cdot \mathrm{s} / \mathrm{kg}]$ & $0.27 \pm 0.08^{*}$ & $0.42 \pm 0.11^{\S}$ & $0.24 \pm 0.09$ \\
Ankle plantarflexion $[\mathrm{Nm} \cdot \mathrm{s} / \mathrm{kg}]$ & $0.33 \pm 0.13^{*}$ & $0.15 \pm 0.16^{\S}$ & $0.62 \pm 0.27$ \\
Hip flexion $[\mathrm{Nm} \cdot \mathrm{s} / \mathrm{kg}]$ & $0.03 \pm 0.03$ & $0.03 \pm 0.02$ & $0.04 \pm 0.04$ \\
Ankle dorsiflexion $[\mathrm{Nm} \cdot \mathrm{s} / \mathrm{kg}]$ & $0.03 \pm 0.03^{*}$ & $0.07 \pm 0.02^{\S}$ & $0.02 \pm 0.02$ \\
\hline mean \pm standard deviation, *: vs Conditon TV $(\mathrm{p}<0.05),{ }^{\S}: \mathrm{vs}$ Condition TM $(\mathrm{p}<0.05)$ &
\end{tabular}

with the collected marker coordinates. We hypothesized that no energy was lost by deformity of the segments as well as conflict and compression of the joints in this rigid-body link model. Joint center coordinates, COG locations in each segment or the whole body, segment angle, segment angular velocity, joint angle and joint moment were calculated using Bodybuilder software (Vicon, Oxford, UK) and the data of the marker coordinates, data of ground reaction force, body height and weight, and coefficients of each body segment inertia according to the method of Okada et al. ${ }^{12}$.

Calculation of mechanical energy transfer between body segments requires raw data regarding mechanical power, the joint force $(\mathrm{F})$ and joint moment $(\mathrm{M})$ related with the observed movement (Fig. 1). According to the Newton's third law, the force and the moment of force acting on joints are equal and are in opposite directions. Because both the force and the moment of force act on the joint centers of both the proximal and distal segments, the velocity of segment $T$ in joint $k$ is estimated to be identical to the velocity of segment $S$ in joint $k$ in both magnitude and direction, resulting in counterbalancing of energy changes caused by joint forces. Thus, we assumed that the joint force transfers energy from one segment to the other but does not influence the total mechanical energy of the body. Joint moments are not counterbalanced because the angular velocities of the segments are generally not equal to each other. Thus, only the moments of force across joints are related with the increase and decrease of total mechanical energy $\sqrt{13,14}$. In this study, energy changes caused by joint moments (moment power) between the thorax, pelvis, thigh and shank segments were individually calculated as the mechanical energy flow. The work done by the joint moment in each segment was calculated by integrating the moment power according to time. Average power was calculated by dividing the work by the time taken in each phase. In this study, the expression of the proximal or the distal portions of the segments was based on the distance from the top or the bottom.

Initiation of the motion was defined as the last transition from negative to positive angular velocities before the ocv- currence of the maximum trunk forward tilt angular velocity. Termination of the motion was defined as the first transition from negative to positive angular velocities after the occurrence of the minimum trunk forward tilt angular velocity. Buttocks-off was defined as the instant the vertical vector of the ground reaction force of the buttocks was less than $10 \mathrm{~N}$. The average of the data collected in three STSs is presented. To avoid the influence of variation of weight among subjects, joint moment was divided by the subject's weight before use in the analyses.

The values were expressed as mean \pm standard deviation. Statistical analyses were executed using the statistical software SPSS ver. 17.0 J for Windows (SPSS). One-way analysis of variance followed by Tukey's multiple comparison test was used for comparing the three conditions in cases of homoscedasticity. Welch's test for equality of means followed by Games-Howell's multiple comparison test was used in cases of heteroscedasticity. The significance level was chosen as less than $5 \%$.

\section{RESULTS}

Table 1 shows the maximum forward tilt angle of each segment under the three different task conditions. The maximum thorax forward tilt angle under condition TM was significantly larger than that under condition $\mathrm{N}(\mathrm{p}<0.05)$, while that under condition TV was significantly smaller than that under condition $\mathrm{N}(\mathrm{p}<0.05)$. The maximum shank forward tilt angle showed no significant difference among the three conditions.

Table 2 shows the joint moment impulses in each segment under the three different task conditions. The impulse of hip joint extension under condition TM was significantly higher than those under conditions $\mathrm{N}$ and TV $(\mathrm{p}<0.05)$. The impulses of knee joint extension and ankle joint dorsiflexion under condition TV were significantly higher than those under conditions $\mathrm{N}$ and TM $(\mathrm{p}<0.05)$.

Figure 2 shows changes in moment power between the thorax and abdomen, abdomen and pelvis, pelvis and thigh, 


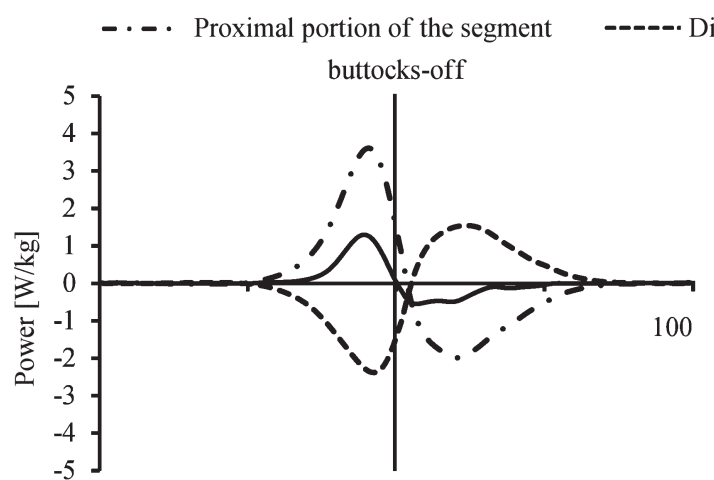

Time [\%]

a) Thorax-Abdomen

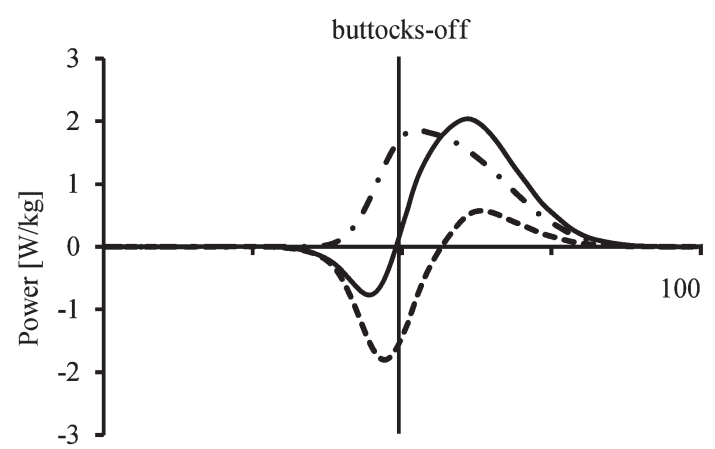

Time [\%]

c) Pelvis-Thigh

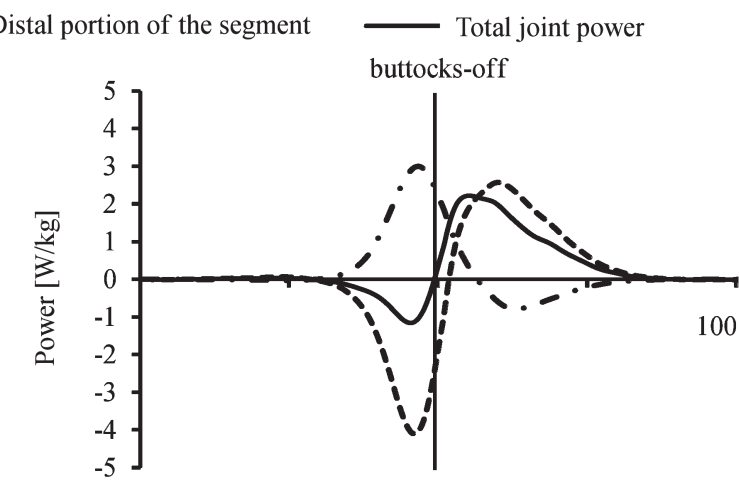

Time [\%]

b) Abdomen-Pelvis

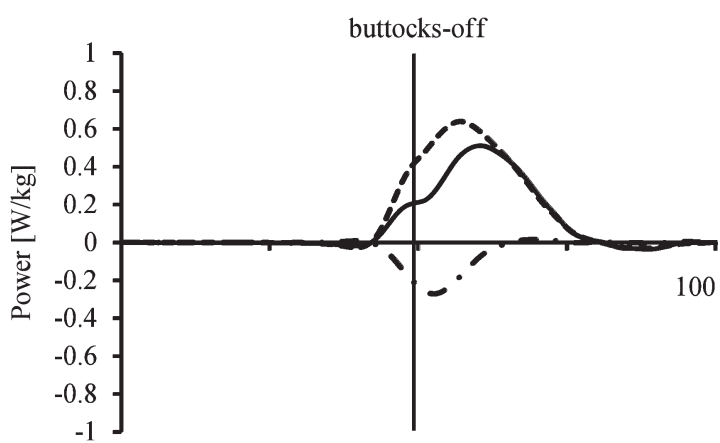

Time [\%]

d) Thigh-Shank

Fig. 2. Changes of muscle power between the thorax and abdomen, abdomen and pelvis, pelvis and thigh, and thigh and shank segments.

Initiation of the motion was defined as $0 \%$ time, buttocks-off was defined as $50 \%$ and termination was defined as $100 \%$ time.

Table 3. Impulses and averages of muscle power under the different conditions

\begin{tabular}{llll}
\hline & Condition N & Condition TV & Condition TM \\
\hline Impulses of muscle power & & & \\
Pelvis distal [W·s $/ \mathrm{kg}$ & $0.54 \pm 0.10^{* \S}$ & $0.31 \pm 0.10^{\S}$ & $0.77 \pm 0.15$ \\
Shank proximal $[\mathrm{W} \cdot \mathrm{s} / \mathrm{kg}]$ & $0.11 \pm 0.06$ & $0.17 \pm 0.06$ & $0.10 \pm 0.07$ \\
Thigh proximal $[\mathrm{W} \cdot \mathrm{s} / \mathrm{kg}]$ & $1.20 \pm 0.17^{*}$ & $0.93 \pm 0.17 \S$ & $1.40 \pm 0.12$ \\
Thigh distal $[\mathrm{W} \cdot \mathrm{s} / \mathrm{kg}]$ & $0.40 \pm 0.19^{*}$ & $0.67 \pm 0.21^{\S}$ & $0.23 \pm 0.11$ \\
Averages of muscle power & & & \\
Pelvis distal $[\mathrm{W} / \mathrm{kg}]$ & $0.88 \pm 0.32$ & $0.85 \pm 0.26$ & $0.66 \pm 0.23$ \\
Shank proximal $[\mathrm{W} / \mathrm{kg}]$ & $0.19 \pm 0.13^{*}$ & $0.38 \pm 0.19^{\S}$ & $0.13 \pm 0.07$ \\
Thigh proximal $[\mathrm{W} / \mathrm{kg}]$ & $0.86 \pm 0.22$ & $0.88 \pm 0.35$ & $0.65 \pm 0.14$ \\
Thigh distal $[\mathrm{W} / \mathrm{kg}]$ & $0.40 \pm 0.21$ & $0.71 \pm 0.34^{\S}$ & $0.22 \pm 0.08$ \\
\hline
\end{tabular}

mean \pm standard deviation, *: vs Conditon TV $(\mathrm{p}<0.05)$, : vs Condition TM $(\mathrm{p}<0.05)$

and thigh and shank segments. During trunk forward tilt, positive moment power in the proximal portions of the pelvis, and negative moment power in the distal portions of the thorax and pelvis were generated. Moreover, positive mo- ment power in the proximal and distal portions of the thigh and negative moment power in the proximal portions of the shank were generated. After buttocks-off, positive moment power in the distal portions of the thorax, pelvis, and thigh, 
and negative moment power in the proximal portions of the pelvis, thigh and shank were generated.

Table 3 shows the impulses of moment power under different conditions. The negative power impulse in the distal portions of the pelvis and positive power impulse in the proximal portions of the thigh under condition TM were significantly higher than those under conditions $\mathrm{N}$ and TV $(p<0.05)$. The average negative power in the distal portions of the pelvis and average positive power in the proximal portions of the thigh showed no significant differences among the three conditions.

The average negative power in the proximal portions of the shank under condition TV was significantly higher than those under conditions $\mathrm{N}$ and TM $(\mathrm{p}<0.05)$, while the negative power impulse in the proximal portions of the shank showed no significant differences among the three conditions. The average positive power in the distal portions of the thigh showed no significant differences among the three conditions, whereas the impulse of positive power in the distal portions of the thigh under condition TV was significantly higher than those under conditions $\mathrm{N}$ and TM $(\mathrm{p}<0.05)$.

\section{DISCUSSION}

STS motion is a postural change from sitting to standing. In STS, COG shifts upward rapidly after shifting forward and downward ${ }^{15}$. Thus, total mechanical energy is increased at the motion termination compared with that at the motion initiation, because COG is elevated in the posture change from sitting to standing. This increased work is done in STS because the change in total mechanical energy is equivalent to the work done by the body. The source of this work is the joint moment acting on each joint since no external force is applied during STS. This suggests the importance of efficiency of the work performed by the joint moment, because energy generated by these works is used for moving body segments. Muscle is the main tissue that provides energy to the body. It also plays a role in storing energy in elastic tissues such as the muscle and tendon by decreasing energy expenditure through eccentric contraction. Robertson et al. 10 demonstrated that the role of joint moment is to transfer energy from one segment to the other, saving energy and decreasing physiological energy expenditure. On the other hand, joint force acting on the joint also transfers energy from one segment to another, but it does not affect the total mechanical energy of the body. Joint moments are associated with changes in energy levels in the body. Therefore, we focus on and discuss the moment power that is related to the energy changes provided by joint moment.

First, we discuss how the mechanical energy flow is generated in STS. We observed positive moment power in the proximal portions of the abdomen, pelvis and thigh, and negative moment power in the distal portions of the thorax, abdomen and pelvis during the movement from initiation of the trunk forward tilt movement to buttocks-off. Muscles in those segments are considered to act to transfer energy when connected segments are rotating in the same direction 17 . We observed that mechanical energy was transferred from the thorax to the abdomen, pelvis and thigh because the seg- ments of thorax, abdomen and pelvis rotated in the same direction during the trunk forward tilt. This indicates that mechanical energy was transferred from the thorax to the thigh via muscles to generate the mechanical energy for the thigh during the trunk forward tilt movement. We consider the magnitude of the negative moment power impulse in the distal portions of the pelvis is stored in the elastic tissues of the hip joint extensors, where it is used to accomplish the task of buttocks-off. In addition, we observed positive power in the distal portions of the thigh and negative power in the proximal portions of the shank during the movement from around buttocks-off to the maximum shank forward tilt. These show the transfer of mechanical energy from the shank to the thigh. That is, the negative moment power in the proximal portions of the shank is kinematic energy generated by shank forward tilt movement which occurs around buttocks-off and is then stored in the elastic tissues of the muscles that extend the knee. The moment power appears to enhance the efficiency of knee extensors. These findings suggest that energy efficiency in STS is enhanced by energy absorption in hip extensors by trunk forward tilt movement as well as by energy absorption in knee extensors by shank forward tilt movement, and the energy transfers to the thigh.

Here, we discuss the influence of trunk forward tilt. In this study, we used condition N under which ordinary STS is performed, condition TV under which the subjects stood up without tilting the trunk, and condition TM under which the subjects stood up while tilting the trunk as forward as possible. These conditions enabled us to examine how the load of lower extremity joints changes in the presence and absence of trunk forward tilt. The maximum thorax forward tilt angle under condition TM was significantly larger than that under condition $\mathrm{N}$ while the angle under condition TV was significantly smaller than that under condition $\mathrm{N}$, confirming that the motion was performed as directed. The impulse of hip extension under condition TM was significantly higher than those under conditions $\mathrm{N}$ and $\mathrm{TV}$, and the impulses of knee extension and ankle dorsiflexion under condition TV were significantly higher than those under conditions N and TM. This result is similar to that of a previous study 18 and indicates that different magnitudes of trunk forward tilt require different moment powers in each joint. Trunk forward tilt is considered to play a role in enhancing the power of lower extremity extensors, especially the knee extensors which move COG upward on a BOS supported by the feet alone ${ }^{15}$. These results indicate that under condition TM, hip extensors and ankle plantar flexors are mainly responsible for the upward shift of COG while under condition TV, and that ankle dorsiflexors and knee extensors are mainly responsible for the forward and upward shifts of COG, respectively.

The negative moment power in the distal portions of the pelvis and impulse of positive power in the proximal portions of the thighs under condition $\mathrm{N}$ were significantly higher than those under condition TV and significantly lower than those under condition TM. We consider the negative power in the distal portions of the pelvis is the mechanical energy that was originally transferred from the pelvis to the elastic tissues of the hip extensors, where the energy was then absorbed. This indicates that tilting the trunk more 
forward increases the absorption of the mechanical energy in the hip extensors. The impulse of hip extension moment was higher under condition TM, indicating that exertion of physiological energy was required by the hip extensors. However, exertion of the physiological energy appeared to be successfully suppressed by utilizing the energy flow during trunk forward tilt, since energy storage increased. Mean values of negative power in the distal portions of the pelvis and positive power in the proximal portions of the thigh showed no significant differences among the three conditions. The mean power value is the work efficiency, i.e. the ability to work, and it indicates that there was no difference in the ability required by the hip joint during STS under any motion condition.

There were no significant differences in the maximum shank forward tilt angle among the different conditions. The impulse of positive power in the distal portions of the thighs under condition TV was significantly higher than those under conditions $\mathrm{N}$ and TM. However, the mean negative power of the proximal portions of the shank under condition TV was significantly higher than those under conditions $\mathrm{N}$ and TM, even though the impulse of negative power did not show any significant differences among the three conditions. Shank forward tilt movement is considered to contribute to moving COG just by the feet on the BOS after buttocksoff 1 . However the maximum shank forward tilt angle did not increase even under condition TV, which stipulated less trunk forward tilt movement. Under condition TV, the absorption of mechanical energy in the hip extensors by trunk forward tilt is low, and the absorption of mechanical energy from shank to the knee extensors was not increased. Based on these findings, we suggest that STS with less trunk forward tilt movement cannot utilize mechanical energy efficiently, and requires greater control of the knee joint and exertion of physiological energy in knee extensors.

In this study, trunk forward tilt and shank forward tilt movements in STS, not only moved COG forward, but also transferred energy to the thigh via muscles generating rotation in the same direction as that of the thigh. This decreased the exertion of physiological energy by muscles in the task of buttocks-off. Most human movements are reactive movements which act in the opposite direction prior to the primary movement. This movement in the opposite direction accumulates mechanical energy in the compulsorily extended elastic element, and the energy is used for the next primary movement 19 . Shank forward tilt appears primarily to be a reactive movement facilitating knee extension, rather than contributing to moving COG forward. Thus, less trunk forward tilt movement results in a requirement of higher ability of shank forward tilt movement. This decline of motor function is expected to decrease the movement efficiency of STS and further increase the load on the muscles. Based on results provided by this study, we anticipate innovative approaches to clinical practices in physical therapy interventions in the future.

A limitation of this study is that we did not measure the extent of elongation of muscles and/or tendons. This does not enable us to conclude that all of the negative moment powers were derived from tendon extension. Further investigation of the detailed behaviour of knee extensors and tendon during STS will be necessary in future studies.

\section{ACKNOWLEDGMENTS}

This is a part of the studies supported by grant-inaid (21700532) by Japan Society for the Promotion of Science (JSPS). We hereby express our sincere appreciation to Dr Yoshihiro Ehara at Niigata University of Health and Welfare for his advice and guidance throughout this study.

\section{REFERENCES}

1) Hodge E, Banowsky L, Novick A, et al.: Alternative immunosuppressive strategies in the management of recipients of living related renal transplants. Transplant Proc, 1989, 21: 1609-1614. [Medline]

2) Riley PO, Krebs DE, Popat RA: Biomechanical analysis of failed sit-tostand. IEEE Trans Rehabil Eng, 1997, 5: 353-359. Medline CrossRef

3) Pai YC, Rogers MW: Control of body mass transfer as a function of speed of ascent in sit-to-stand. Med Sci Sports Exerc, 1990, 22: 378-384. [Medline]

4) Nyberg L, Gustafson Y: Patient falls in stroke rehabilitation. Stroke, 1995, 26: 838-842. Medline CrossRef

5) Hughes MA, Weiner DK, Schenkman ML, et al.: Chair rise strategies in the elderly. Clin Biomech (Bristol, Avon), 1994, 9: 187-192. [CrossRef]

6) Shumway-Cook A, Woollacott M: Control of normal mobility. In: Motor control translating tesearch into clinical practice 4th ed. Baltimore: Williams and Wilkins, 2010, pp 315-347.

7) Cavanagh PR, Kram R: The efficiency of human movement-a statement of the problem. Med Sci Sports Exerc, 1985, 17: 304-308. Medline

8) Williams KR, Cavanagh PR: Relationship between distance running mechanics, running economy, and performance. J Appl Physiol, 1987, 63: 1236-1245. Medline

9) Yu B, Holly-Crichlow N, Brichta P, et al.: The effects of the lower extremity joint motions on the total body motion in sit-to-stand movement. Clin Biomech (Bristol, Avon), 2000, 15: 449-455. [Medline [CrossRef

10) Kurabayashi J, Mochimaru M, Kouchi M: Validation of the estimation methods for the hip joint center. J Soc Biomechanism, 2003, 27: 29-36 (In Japanese). [CrossRef

11) Andriacchi TP, Galante JO, Fermier RW: The influence of total knee replacement design on walking and stair-climbing. J Bone Joint Surg Am, 1982, 64: 1328-1335. [Medline

12) Okada H, Ae M, Fujii N, et al.: Body segment inertia properties of Japanese elderly. Biomechanism, 1996, 13: 125-139 (In Japanese).

13) Robertson DG: Energy, work, and power. In: Research Methods in Biomechanics. Champaign: Human Kinetics, 2004, pp125-144.

14) Winter DA: Mechanical work, energy, and power. In: Biomechanics and motor control of human movement.4th ed. Hoboken: John Wiley \& Sons, 2009, pp 139-175.

15) Kelly DL, Dainis A, Wood GK: Mechanics and muscular dynamics of rising from a seated position. In: Biomechanics. Baltimore: University Park Press, 1976, pp 127-134

16) Gordon D, Winter DA: Mechanical energy generation, absorption transfer amongst segments during walking. J Biomech, 1980, 13: 845-854. Medine CrossRef

17) Aleshinsky SY: An energy 'sources' and 'fractions' approach to the mechanical energy expenditure problem: $V$. The mechanical energy expenditure reduction during motion of the multi-link system. J Biomech, 1986, 19: 311-315. Medline [CrossRef

18) Doorenbosch CA, Harlaar J, Roebroeck ME, et al.: Two strategies of transferring from sit-to-stand: the activation of monoarticular and biarticular muscles. J Biomech, 1994, 27: 1299-1307. [Medline CrossRef

19) Fukashiro $S$, Itoh $M$, Ichinose $Y$, et al.: Untrasonography gives directly but noninvasively elastic characteristic of human tendon in vivo. Eur J Appl Physiol Occup Physiol, 1995, 71: 555-557. [Medline] [CrossRef 